Communications in Physics, Vol. 19, No. 4 (2009), pp. 205-221

\title{
MULTIPARTITE DISENTANGLEMENT DYNAMICS DUE SIMULTANEOUSLY TO AMPLITUDE DAMPING AND PHASE DAMPING
}

\author{
NGUYEN BA AN \\ Institute of Physics, VAST
}

\begin{abstract}
We present a detailed analysis on disentanglement dynamics of multiqubit GHZ-type states whose qubits are remotely located in absence of any mutual interactions. The dynamics is thus induced by independent local environments surrounding each qubit. It has recently been known that if each qubit is subjected solely to the phase damping then the state's entanglement vanishes asymptotically in time and if only the amplitude damping is active then the state's entanglement may vanish suddenly in certain parameter subspace. In this paper, we shall show that a combined action of both the phase damping and the amplitude damping will force the state's entanglement to always vanish suddenly in the entire parameter space. Furthermore, we shall prove that by proper local operations such a finite-time disentanglement can be avoided for whatever state's parameters, no matter the phase damping and the amplitude damping act severally or in combination.
\end{abstract}

\section{INTRODUCTION}

It is the nonlocal correlation between the subsystems comprising a quantum system that makes quantum entanglement (or, simply entanglement, for short) so fascinating and having no classical counterparts. Many surprising quantum protocols/algorithms based on entanglement have been proposed so far. Superdense coding [1], quantum teleportation [2], quantum key distribution [3], quantum secret sharing [4], prime factorization [5], quantum search [6], etc. are most profound examples. Recently, the issues such as multipartite entanglement, disentanglement dynamics, combined action of different types of noise sources and controlling disentanglement dynamics have attracted special attention among the quantum physics community. Multipartite entanglement is necessary for quantum secure network communication and scalable quantum computation. Disentanglement dynamics differs very much from decoherence and is inevitable in realistic circumstances due to interactions with always existing environments. Combined action of noises of distinct types is more likely to happen in practice than separate actions of them. And, controlling disentanglement dynamics is highly desirable from the application point of view because one needs to maintain as long as possible the initial entanglement amount to perform a quantum task.

Multipartite disentanglement dynamics has recently been intensively studied by a number of authors [7-17], focusing just on particular effects of an individual noise source. The collective effect of simultaneous action of two different noise sources has been explored in Ref. [18] but only for a bipartite model, the simplest possible composite system. In this work we shall touch upon all the important issues mentioned above by investigating 
disentanglement dynamics of the $N$-qubit GHZ-type state

$$
\left|\Phi_{N}\right\rangle=\alpha|0\rangle^{\otimes N}+\beta|1\rangle^{\otimes N}
$$

with nonzero $\alpha, \beta \in \mathcal{C}$ and $|\alpha|^{2}+|\beta|^{2}=1$, a very powerful quantum resource which has already been generated in the lab for $N$ up to 10 [19] and suitable to a good deal of application (see, e.g., [20-23]), under simultaneous local influence of two typical quantum damping channels [24] at zero temperature, the phase damping and the amplitude damping ones. Here by qubits we mean two-level atoms with one ground state denoted by $|0\rangle$ and one excited state denoted by $|1\rangle$. We assume a quite reasonable experimental scenario in which the $N$ qubits are shared among $N$ remote parties so that each qubit interacts only with its own environment and there are no direct or indirect interactions at all between any qubit pair. Since there exists nonlocal "spooky" correlation between the qubits, even local environments can lead to far-reaching consequences of the state's global properties such as entanglement. We are interested in the time it takes for state $\left|\Phi_{N}\right\rangle$ to be fully disentangled, i.e., to become completely separable. In Sec. II we outline a description of the state evolution governed by local qubit-environment interactions using the so-called operatorsum representation in terms of Kraus operators [25]. Sec. III presents a detailed analysis of disentanglement dynamics of $\left|\Phi_{N}\right\rangle$, with emphasis on an interesting phenomenon named finite-time disentanglement (FTD), which is also called entanglement sudden death in the literatures [26-38]. In Sec. IV, a method using certain local operations on the initial state $\left|\Phi_{N}\right\rangle$ is introduced to transform it to another state which possesses the same entanglement amount as $\left|\Phi_{N}\right\rangle$, but, unlike $\left|\Phi_{N}\right\rangle$, never suffers from FTD. Since disentanglement time does not fully characterize usefulness of an entangled state we also analyze in this section the initial time evolution of both entanglement and fidelity of $\left|\Phi_{N}\right\rangle$ as well as of the states obtained from $\left|\Phi_{N}\right\rangle$ by the local operations. Finally, we conclude in Sec. V.

\section{THE OPERATOR-SUM REPRESENTATION}

Suppose that initially a qubit $j$ is in a state $\rho^{j}(0)$, which may be pure or mixed, and its surrounding environment $E^{j}$ is in the vacuum state $|0\rangle_{E^{j}}$. Let at $t>0$ the total system "qubit plus environment" evolves together in time via a unitary operator $U_{j E^{j}}(t)$ :

$$
\rho^{j}(0) \otimes|0\rangle_{E^{j} E^{j}}\langle 0| \rightarrow \rho^{j E^{j}}(t)=U_{j E^{j}}(t)\left(\rho^{j}(0) \otimes|0\rangle_{E^{j} E^{j}}\langle 0|\right) U_{j E^{j}}^{+}(t) .
$$

Since we are interested in the qubit dynamics we need trace out $\rho^{j E^{j}}(t)$ over the degrees of freedom of the uncontrollable environment. This can be expressed as action of a superoperator $\mathcal{S}$ on $\rho^{j}(0)$ as

$$
\mathcal{S} \rho^{j}(0)=\rho^{j}(t)=\operatorname{Tr}_{E^{j}}\left[\rho^{j E^{j}}(t)\right]=\sum_{n} K_{n}^{j}(t) \rho^{j}(0) K_{n}^{j}(t)^{+}
$$

where

$$
K_{n}^{j}(t)={ }_{E^{j}}\left\langle n\left|U_{j E^{j}}(t)\right| 0\right\rangle_{E^{j}}
$$


with $\left\{|n\rangle_{E^{j}}\right\}$ an orthogonal basis for the environment associated with qubit $j$, are called Kraus operators [25] which satisfy the trace-preserving condition

$$
\sum_{n} K_{n}^{j}(t)^{+} K_{n}^{j}(t)=I
$$

for all time $t$. Equation (3) is referred to as the operator-sum representation of the superoperator $\mathcal{S}$ which is in many cases very convenient to obtain the desired $\rho^{j}(t)$ directly from $\rho^{j}(0)$. The Kraus operators themselves can be derived from the corresponding unitary operator by means of Eq. (4).

Two typical and useful quantum damping channels [24] for qubits are the phase damping channel and the amplitude damping channel. A combined action of both the channels on qubit $j$ can be described by the following quantum map (corresponding to transformations governed by the underlying unitary operator $U_{j E^{j}}$ )

$$
U_{j E^{j}}:\left\{\begin{array}{rll}
|00\rangle_{j E^{j}} \rightarrow & |00\rangle_{j E^{j}}, \\
|10\rangle_{j E^{j}} \rightarrow & \sqrt{\left(1-p_{j}\right)\left(1-P_{j}\right)}|10\rangle_{j E^{j}}+\sqrt{p_{j}\left(1-P_{j}\right)}|11\rangle_{j E^{j}} \\
& +\sqrt{\left(1-p_{j}\right) P_{j}}|02\rangle_{j E^{j}}+\sqrt{p_{j} P_{j}}|03\rangle_{j E^{j}} .
\end{array}\right.
$$

Physically, this map implies no changes in case the qubit is in its ground state $|0\rangle_{j}$. But, when the qubit is in its excited state $|1\rangle_{j}$, it may either stay there without doing anything with probability $\left(1-p_{j}\right)\left(1-P_{j}\right)$ or with scattering the environment to state $|1\rangle_{E^{j}}$ with probability $p_{j}\left(1-P_{j}\right)$, or it may jump down to its ground state and transfers its energy to the environment by exciting it to state $|2\rangle_{E^{j}}$ with probability $\left(1-p_{j}\right) P_{j}$ or to state $|3\rangle_{E^{j}}$ with probability $p_{j} P_{j}$. In Eq. (6) $p_{j}$ and $P_{j}$ are the transition probabilities due to the phase damping and the amplitude damping, respectively, and time is parameterized though them as

$$
\begin{aligned}
& p_{j} \equiv p_{j}(t)=1-e^{-\gamma_{j} t}, \\
& P_{j} \equiv P_{j}(t)=1-e^{-\Gamma_{j} t},
\end{aligned}
$$

with $\gamma_{j}$ and $\Gamma_{j}$ the decay rates of qubit $j$ associated with the corresponding damping channel. If during the course of evolution we could monitor the environment and at a given time measure it in the basis $\left\{|0\rangle_{E^{j}},|1\rangle_{E^{j}},|2\rangle_{E^{j}},|3\rangle_{E^{j}}\right\}$, we would be able to infer the qubit state at that time. In case the environment is uncontrollable we should average over its states. Using the map (6) in Eq. (4) we have derived the four underlying Kraus operators which in the qubit computational basis $\left\{|0\rangle_{j},|1\rangle_{j}\right\}$ are of the form

$$
\begin{aligned}
K_{0}^{j} & =\left(\begin{array}{cc}
1 & 0 \\
0 & \sqrt{\left(1-p_{j}\right)\left(1-P_{j}\right)}
\end{array}\right), \\
K_{1}^{j} & =\left(\begin{array}{cc}
0 & 0 \\
0 & \sqrt{p_{j}\left(1-P_{j}\right)}
\end{array}\right), \\
K_{2}^{j} & =\left(\begin{array}{cc}
0 & \sqrt{\left(1-p_{j}\right) P_{j}} \\
0 & 0
\end{array}\right), \\
K_{3}^{j} & =\left(\begin{array}{cc}
0 & \sqrt{p_{j} P_{j}} \\
0 & 0
\end{array}\right) .
\end{aligned}
$$


It is straightforward to check that the above operators $K_{n}^{j}$ satisfy the condition (5) for any $p_{j}, P_{j}$ (i.e., for all time) and that they reproduce the right expressions of Kraus operators for the amplitude damping or the phase damping [36,37] when $p_{j}=0$ or $P_{j}=0$.

According to the experimental scenario mentioned in Introduction, there are no common environments to which some qubits may be coupled, a situation that may result in so-called decoherence-free subspaces $[39,40]$. There is only a local environment for each qubit within which the qubit is experienced at the same time by both the phase damping and the amplitude damping. Thus, the evolution of any ensemble of $N$ distant qubits is determined by

$$
\rho^{12 \ldots N}(t)=\sum_{\left\{n_{j}\right\}=0}^{3} \mathcal{K}_{n_{1} n_{2} \ldots n_{N}}^{12 \ldots N}(t) \rho^{12 \ldots N}(0) \mathcal{K}_{n_{1} n_{2} \ldots n_{N}}^{12 \ldots N}(t)^{+}
$$

with

$$
\mathcal{K}_{n_{1} n_{2} \ldots n_{N}}^{12 \ldots N}(t)=K_{n_{1}}^{1}(t) \otimes K_{n_{2}}^{2}(t) \otimes \cdots \otimes K_{n_{N}}^{N}(t)
$$

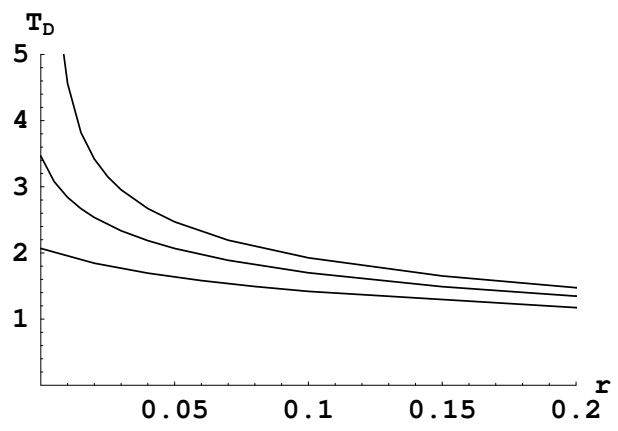

Fig. 1. The dimensionless disentanglement time $T_{D}=\Gamma t_{D}$ as a function of $r=$ $\gamma / \Gamma$ for $\Gamma_{j}=\Gamma, \gamma_{j}=\gamma, N=6$ and $s=|\beta / \alpha|=0.8,1.1$ and 1.5 (from top to bottom). $T_{D}$ is infinite (no FTD) at $r=0$ for $s=0.8$ but it is finite (FTD exists) at all $r$ including $r=0$ for $s=1.1,1.5$. Generally, for a fixed $N, T_{D}$ decreases with increasing $r$ or/and $s$.

\section{DISENTANGLEMENT DYNAMICS}

The state $\left|\Phi_{N}\right\rangle$ given in Eq. (1) has the explicit form

$$
\left|\Phi_{N}\right\rangle=(\alpha|00 \ldots 0\rangle+\beta|11 \ldots 1\rangle)_{12 \ldots N},
$$

which is a coherent superposition of $N$ " 0 " and $N$ "1". Besides the crucial role played by the state (15) in many problems of quantum communication and quantum computation [20-23], the main reason of choosing it for our investigation here is that this state's disentanglement dynamics has recently been dealt with in Ref. [13] under separate damping channels and we would like to discover new features that may arise when more than one channel act together at the same time.

By virtue of Eqs. (13) and (9) to (12), under simultaneous action of both the phase damping and the amplitude damping channels, the initial pure state $\rho^{\Phi}(0)=\left|\Phi_{N}\right\rangle\left\langle\Phi_{N}\right|=$ 


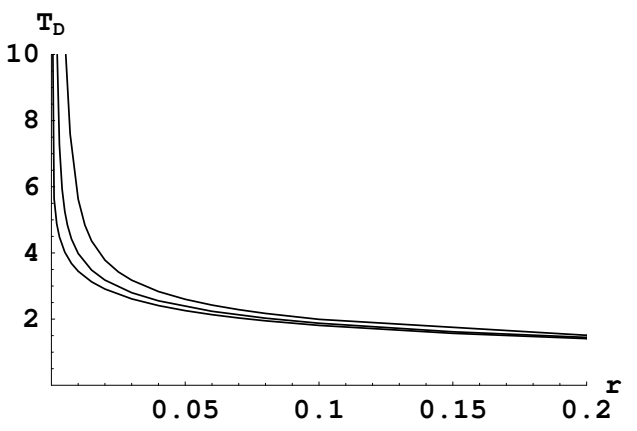

Fig. 2. The dimensionless disentanglement time $T_{D}=\Gamma t_{D}$ as a function of $r=$ $\gamma / \Gamma$ for $\Gamma_{j}=\Gamma, \gamma_{j}=\gamma, s=|\beta / \alpha|=0.9$ and $N=4,10$ and 100 (from top to bottom). $T_{D}$ is infinite (no FTD) at $r=0$ since $s=0.9<1$. For $r>0$, FTD occurs and $T_{D}$ decreases with increasing $r$ or/and $N$.

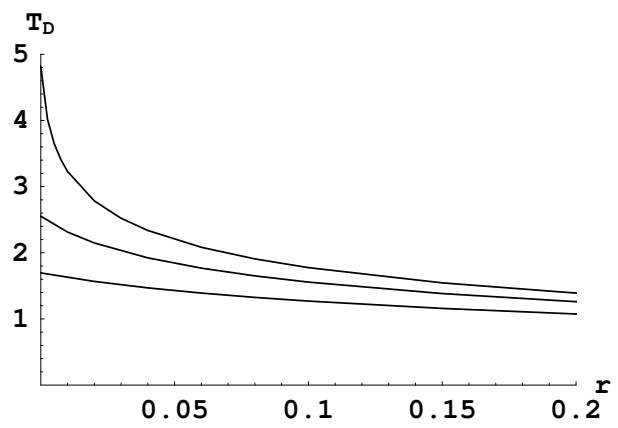

Fig. 3. The dimensionless disentanglement time $T_{D}=\Gamma t_{D}$ as a function of $r=$ $\gamma / \Gamma$ for $\Gamma_{j}=\Gamma, \gamma_{j}=\gamma, s=|\beta / \alpha|=1.5$ and $N=4,10$ and 100 (from bottom to top). Since $s=1.5>1$ FTD occurs for all $r$ including $r=0$. In this case $T_{D}$ decreases with $r$ but increases with $N$.

$|\alpha|^{2}|00 \ldots 0\rangle\left\langle 00 \ldots 0\left|+\alpha \beta^{*}\right| 00 \ldots 0\right\rangle\left\langle 11 \ldots 1\left|+\beta \alpha^{*}\right| 11 \ldots 1\right\rangle\left\langle\left. 00 \ldots 0|+| \beta\right|^{2} \mid 11 \ldots 1\right\rangle\langle 11 \ldots 1|$ evolves into a mixed state

$$
\begin{aligned}
\rho^{\Phi}(t)= & a|00 \ldots 0\rangle\langle 00 \ldots 0|+b| 00 \ldots 0\rangle\left\langle 11 \ldots 1\left|+b^{*}\right| 11 \ldots 1\right\rangle\langle 00 \ldots 0| \\
& +\sum_{\left\{a_{j}\right\}} b_{a_{1} a_{2} \ldots a_{N}}\left|a_{1} a_{2} \ldots a_{N}\right\rangle\left\langle a_{1} a_{2} \ldots a_{N}\right|
\end{aligned}
$$




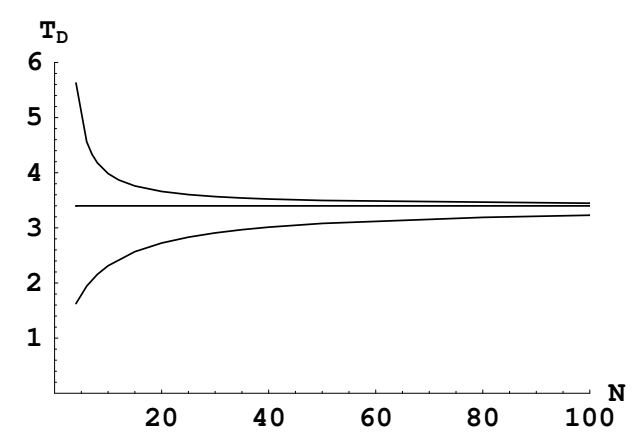

Fig. 4. The scaling of the dimensionless disentanglement time $T_{D}=\Gamma t_{D}$ for state $\left|\Phi_{N}\right\rangle$ with the qubit number $N$. The parameters used are $\Gamma_{j}=\Gamma, \gamma_{j}=\gamma$, $r=\gamma / \Gamma=0.01$ and $s=|\beta / \alpha|=0.9,1$ and 1.5 (from top to bottom). In the large- $N$ limit $T_{D}$ ceases to depend on $s$.

where the sum over $\left\{a_{j}\right\}$ runs for all possible $a_{1}, a_{2}, \ldots, a_{N} \in\{0,1\}$ except $a_{1}=a_{2}=\ldots=$ $a_{N}=0$ (whose contribution is included in $a$ for convenience),

$$
\begin{aligned}
a & =|\alpha|^{2}+|\beta|^{2} \prod_{j=1}^{N}\left(1-e^{-\Gamma_{j} t}\right), \\
b & =\alpha \beta^{*} e^{-\sum_{j=1}^{N}\left(\gamma_{j}+\Gamma_{j}\right) t / 2}, \\
b_{a_{1} a_{2} \ldots a_{N}} & =|\beta|^{2} \prod_{j=1}^{N}\left[\overline{a_{j}}\left(1-e^{-\Gamma_{j} t}\right)+a_{j} e^{-\Gamma_{j} t}\right]
\end{aligned}
$$

with $\overline{a_{j}}=1-a_{j}$.

As no fully satisfactory methods exist to quantify entanglement of an arbitrary multipartite state, various entanglement measures such as generalized concurrence [41], negativity [42,43], Meyer-Wallach measure [44], geometric measure [45], etc. have been invoked to. To be concrete, we use negativity in this work. For state $\rho$ of a system of $N$ parties, the negativity associated with a bipartition $k \mid N-k$ is defined as $\mathcal{N}_{k}=2\left|\sum_{n} \lambda_{n}\right|$, where $\lambda_{n}$ are the negative eigenvalues of $\rho^{T_{k}}$, the partial transpose of $\rho$ with respect to the concerned bipartition. Then, from $\mathcal{N}_{k}=0$ it follows that $\rho^{T_{k}}>0$, i.e., $\rho$ is a positive partial transpose (PPT) state. Nevertheless, this does not generally imply separability of $\rho$. In fact, $\rho^{T_{k}}>0$ guarantees separability only in Hilbert spaces of dimensions $2 \times 2$ or $2 \times 3$, but for higher dimensions there may exist states that are entangled and, at the same time, PPT. Such PPT entangled states are called bound entangled ones because they cannot be distilled [46]. Therefore, negativity cannot quantify possible entanglement in dimensions higher than six. However, for states like $\rho^{\Phi}(t)$ calculation of negativities reduces to a problem of dimension $2 \times 2$ so for $\rho^{\Phi}(t)$ null negativity ensures separability in the corresponding bipartition. Furthermore, $\rho^{\Phi}(t)$ belong to a class of states whose partial transposes have at most one negative eigenvalue. For such states the expression for $\mathcal{N}_{k}$ simplifies to

$$
\mathcal{N}_{k}=2 \max \left\{0,-\lambda_{k}\right\},
$$


with $\lambda_{k}$ the minimum eigenvalue of $\rho^{T_{k}}$.

Return to state (15) and consider a bipartition $k \mid N-k$ of it. The partial transpose $\rho^{\Phi}(t)^{T_{k}}$ corresponding to the bipartition has the form

$$
\begin{aligned}
\rho^{\Phi}(t)^{T_{k}}= & a|00 \ldots 0\rangle\langle 00 \ldots 0|+b| k\rangle\left\langle\bar{k}\left|+b^{*}\right| \bar{k}\right\rangle\langle k| \\
& +\sum_{\left\{a_{j}\right\}} b_{a_{1} a_{2} \ldots a_{N}}\left|a_{1} a_{2} \ldots a_{N}\right\rangle\left\langle a_{1} a_{2} \ldots a_{N}\right|,
\end{aligned}
$$

with $|k\rangle=\left|c_{1} c_{2} \ldots c_{N}\right\rangle$ and $|\bar{k}\rangle=\left|\overline{c_{1} c_{2}} \ldots \overline{c_{N}}\right\rangle$ where among the $N$ values of $\left\{c_{j} ; j=\right.$ $1,2, \ldots, N\}$ there are $k$ " 1 " and $(N-k)$ " 0 ". The minimum eigenvalue $\lambda_{k}$ of $\rho^{\Phi}(t)^{T_{k}}$ can be derived as

$$
\lambda_{k}=\frac{1}{2}\left(b_{k}+b_{\bar{k}}-\sqrt{\left(b_{k}+b_{\bar{k}}\right)^{2}+4\left(|b|^{2}-b_{k} b_{\bar{k}}\right)}\right),
$$

where

$$
b_{k}=|\beta|^{2} \prod_{j=1}^{N}\left[\overline{c_{j}}\left(1-e^{-\Gamma_{j} t}\right)+c_{j} e^{-\Gamma_{j} t}\right]
$$

and

$$
b_{\bar{k}}=|\beta|^{2} \prod_{j=1}^{N}\left[c_{j}\left(1-e^{-\Gamma_{j} t}\right)+\overline{c_{j}} e^{-\Gamma_{j} t}\right] .
$$

Clearly from Eq. (20), the condition for $\mathcal{N}_{k}$ to vanish is $\lambda_{k}=0$ which, by virtue of Eq. $(22)$, is satisfied by

$$
|b|^{2}-b_{k} b_{\bar{k}}=0
$$

A remarkable property is that, although $b_{k}$ and $b_{\bar{k}}$ depend explicitly on $k$ (through $\left\{c_{j}, \overline{c_{j}}\right\}$ ), their product, by virtue of $c_{j} \overline{c_{j}}=0$ and $c_{j}^{2}+{\overline{c_{j}}}^{2}=1$ for any $j$, can be proved to be

$$
b_{k} b_{\bar{k}}=|\beta|^{4} \prod_{j=1}^{N}\left[\left(1-e^{-\Gamma_{j} t}\right) e^{-\Gamma_{j} t}\right]
$$

which displays no $k$-dependence at all. Thus, using Eqs. (18) and (26) in Eq. (25) yields

$$
e^{-t \sum_{j=1}^{N} \Gamma_{j}}\left[e^{-t \sum_{j=1}^{N} \gamma_{j}}-\left|\frac{\beta}{\alpha}\right|^{2} \prod_{j=1}^{N}\left(1-e^{-\Gamma_{j} t}\right)\right]=0 .
$$

The $k$-independence of the condition (27) means that all the $\mathcal{N}_{k}$ are going to vanish at the same time despite each of them may undergo its own different transient evolution. It has been proved in Ref. [13] that, when all the possible negativities vanish, the corresponding state becomes completely separable. Therefore, the time at which the state $\left|\Phi_{N}\right\rangle$ loses its entire entanglement is the solution of Eq. (27). Let us first consider particular cases: a purely dephasing process and a purely dissipative one.

For a purely dephasing process (i.e., $\Gamma_{j}=0 \forall j$ ), the condition (27) reduces to

$$
e^{-t \sum_{j=1}^{N} \gamma_{j}}=0,
$$


which is satisfied only in the limit $t \rightarrow \infty$, implying an entanglement asymptotic vanishing with the individual decay rates $\gamma_{j}$ being added. This effect of additivity of decay rates is similar to decoherence processes.

For a purely dissipative process (i.e., $\gamma_{j}=0 \forall j$ ), the condition (27) reduces to

$$
e^{-t \sum_{j=1}^{N} \Gamma_{j}}\left[1-\left|\frac{\beta}{\alpha}\right|^{2} \prod_{j=1}^{N}\left(1-e^{-\Gamma_{j} t}\right)\right]=0 .
$$

In this case there appear two regimes of disentanglement, depending on the parameters. For $|\alpha| \geq|\beta|$ the value of the expression in the square brackets decreases in time but remains always positive. Hence, the condition (29) is equivalent to

$$
e^{-t \sum_{j=1}^{N} \Gamma_{j}}=0
$$

which is again satisfied in the limit $t \rightarrow \infty$, with the individual decay rates $\Gamma_{j}$ being added too. However, for $|\alpha|<|\beta|$ the value of the expression in the square brackets may vanish in a finite time $t<\infty$. Then the condition (29) is equivalent to

$$
1-\left|\frac{\beta}{\alpha}\right|^{2} \prod_{j=1}^{N}\left(1-e^{-\Gamma_{j} t}\right)=0,
$$

which implies FTD [26-38] with the individual decay rates $\Gamma_{j}$ not being simply added anymore. Such FTD effect is absolutely distinct from decoherence processes. The above particular results for purely dephasing and purely dissipative processes coincide with those obtained in Ref. [13] for identical local environments.

Now, what will happen if the qubits are experienced at the same time by both the noise sources? Since as time grows the first term in the square brackets of Eq. (27) decreases from 1 to 0 , while the second term increases from 0 to $|\beta / \alpha|^{2}$, the two will inevitably intersect at a finite time for whatever values of $\alpha$ and $\beta$. In this case, the condition (27) is equivalent to

$$
e^{-t \sum_{j=1}^{N} \gamma_{j}}-\left|\frac{\beta}{\alpha}\right|^{2} \prod_{j=1}^{N}\left(1-e^{-\Gamma_{j} t}\right)=0,
$$

which is clearly by no ways related to $\exp \left[-t \sum_{j=1}^{N}\left(\gamma_{j}+\Gamma_{j}\right]\right.$, i.e., the individual decay rates $\gamma_{j}, \Gamma_{j}$ never add. Physically, this result means that a combined action of both the phase damping and the amplitude damping allows only one regime, the FTD one, for $\left|\Phi_{N}\right\rangle$ to disentangle. Of somewhat unexpected surprise is the role played by the phase damping. When it acts alone no FTD occurs. But when it acts together with the amplitude damping it enhances the effect of FTD in the sense that FTD occurs for any values of $\alpha$ and $\beta$, but not only for $|\alpha|<|\beta|$ as in the case when only the amplitude damping acts. As mentioned above, whenever FTD occurs the individual decay rates cease to be additive. The most pronounced breakdown of additivity of decay rates can be seen in the parameter domain with $|\alpha| \geq|\beta|$. In this domain, each of the noise sources alone only cause infinite-time disentanglement, but under their combined action FTD becomes compulsory! 
In Ref. [13] the local environments are identical, i.e., $\gamma_{j}=\gamma$ and $\Gamma_{j}=\Gamma \forall j$. Here we consider a more general situation with nonidentical environments, i.e., $\gamma_{i} \neq \gamma_{j}$ and $\Gamma_{i} \neq \Gamma_{j}$ for $i \neq j$. This allows us to further clarify the role of local noises with respect to the occurrence of FTD. From the above analysis it can be verified that the necessary constraints to always trigger FTD are

$$
\prod_{j=1}^{N} \Gamma_{j}>0
$$

and

$$
\sum_{j=1}^{N} \gamma_{j}>0 .
$$

The constraint (33) demands all $\Gamma_{j}$ be greater than zero, i.e., all the qubits should be subjected to the amplitude damping in their local environments. Should any qubit be "liberated" from the amplitude damping, the whole qubits' system will disentangle asymptotically in an infinite time. On the other hand, the constraint (34) just requires at least one qubit to be under the local phase damping. Of course, however, the greater the number of qubits being under local phase dampings the sooner the time of FTD occurrence.

To visualize the influence of all the involved parameters on the disentanglement dynamics, let us assume for simplicity $\gamma_{j}=\gamma$ and $\Gamma_{j}=\Gamma$. Then the disentanglement time $t_{D}$ is solution of the equation

$$
e^{-r T_{D}}=s^{2 / N}\left(1-e^{-T_{D}}\right)
$$

in which we have used the following dimensionless notations $T_{D}=\Gamma t_{D}, r=\gamma / \Gamma$ and $s=|\beta / \alpha|$. In Fig. 1 we plot $T_{D}$ as a function of $r$ for a fixed value of $N$ but different values of $s$. If $s \leq 1, T_{D}=\infty$ for $r=0$, i.e., no FTD arises in the absence of phase damping. However, $T_{D}$ becomes finite for $r>0$, i.e., FTD arises in the presence of phase damping. When $s>1$, FTD occurs for whatever value of $r$ including $r=0$. Generally, for a given $N$, the dimensionless disentanglement time $T_{D}$ decreases with increasing $r$ or/and $s$. Figure 2 plots $T_{D}$ versus $r$ for a fixed value of $s<1$ but different values of $N$. There is no FTD for any $N$ if $r=0$ but, if $r>0$, FTD occurs with $T_{D}$ decreasing with increasing $r$ or/and $N$. Figure 3 is the same as Fig. 2 but for $s>1$. In Fig. 3, similar to Fig. 2, $T_{D}$ decreases with increasing $r$, but opposed to Fig. $2, T_{D}$ increases with increasing $N$. It is worth emphasizing that for $r>0$ the scaling of $T_{D}$ with the system size in terms of the qubit number $N$ differs strongly in three regimes $s=1, s<1$ and $s>1$. Namely, there is no $N$-dependence for $s=1$. However, the $N$-dependences are inverse to each other for $s<1$ and $s>1$. Moreover, for $s<1(s>1)$ the scaled disentanglement time $T_{D}$ is bound from below (above) by its value at $s=1$. In other words, in the limit $N \rightarrow \infty$ (large-size limit) $T_{D}$ ceases to depend on $s$. These behaviors are illustrated in Fig. 4 . In the largesize limit $(N \rightarrow \infty)$, only the $r$-dependence sustains and, for $r>0$, the dimensionless disentanglement time is determined by $T_{D}=\ln (1 / X)$ with $X$ the solution of the equation $X^{r}+X=1$. 


\section{CONTROLLING DISENTANGLEMENT DYNAMICS}

If entanglement degrades asymptotically one could distill it when fidelity remains sufficiently high $[47,48]$. Nevertheless, when an entangled state evolves too quickly and suddenly becomes completely separable no distillation protocols can be utilized to restore the irreversible loss of entanglement. FTD has been looked upon as a serious obstacle or even an impasse in using entanglement as a resource for quantum communication and quantum computation. Controlling FTD dynamics is therefore of real necessity. One method to combat FTD is to let subsystems of the considered system interact with common environments to build up possible decoherence-free subspaces [39,40]. This method however does not apply to our experimental scenario in which each qubit experiences only its own local environment, i.e., there are no common environments at all. FTD in cavity QED can be controlled by measurement feedback methods or by driving the system with additional classical fields (see, e.g., $[49,50]$ and the references therein). Quantum error correction (QEC) [51-53] proves to be a powerful method to detect and correct a general error in a single qubit in closed systems. For open systems a channel-adapted QEC scheme has been proposed [54] that allows to correct errors in single qubits due to the amplitude damping as well. Recently, quantum codes adapted for dissipative channels have been investigated in detail [55] with a hope to protect two-qubit entanglement against the amplitude damping. It has been found that generally the quantum codes will not protect a state from FTD. In some cases they can delay FTD but cannot remove it. Worse still, in other cases they may bring about FTD for coded states which are FTD-free when uncoded!

In this section we shall show that just by applying on $\left|\Phi_{N}\right\rangle$ a proper initial set of local operations one can absolutely get rid of FTD. The set of operations is

$$
U(n)=I^{\otimes n} \otimes\left(\sigma_{x}\right)^{\otimes(N-n)},
$$

with $1 \leq n \leq N-1, I$ the $2 \times 2$ identity matrix and $\sigma_{x}$ the Pauli bit-flip operator. We shall provide an explicit proof that, for any $\alpha$ and $\beta$, entanglement of the transformed state

$$
\left|\Psi_{n, N}\right\rangle=U(n)\left|\Phi_{N}\right\rangle=\alpha|0\rangle^{\otimes n}|1\rangle^{\otimes(N-n)}+\beta|1\rangle^{\otimes n}|0\rangle^{\otimes(N-n)}
$$

always vanishes asymptotically (i.e., FTD never happens), no matter the phase damping and the amplitude damping act severally or together.

By virtue of Eqs. (13) and (9) to (12), under simultaneous action of both the phase damping and the amplitude damping channels, the initial pure state $\rho^{\Psi}(0)=$ $\left|\Psi_{n, N}\right\rangle\left\langle\left.\Psi_{n, N}|=| \alpha\right|^{2} \mid 0 \ldots 01 \ldots 1\right\rangle\left\langle 0 \ldots 01 \ldots 1\left|+\alpha \beta^{*}\right| 0 \ldots 01 \ldots 1\right\rangle\left\langle 1 \ldots 10 \ldots 0\left|+\alpha^{*} \beta\right| 1 \ldots 10 \ldots 0\right\rangle\langle 0 \ldots 01 \ldots 1|+$ $|\beta|^{2}|1 \ldots 10 \ldots 0\rangle\langle 1 \ldots 10 \ldots 0|$ evolves into a mixed state

$$
\begin{aligned}
\rho^{\Psi}(t)= & c|0 \ldots 00 \ldots 0\rangle\langle 0 \ldots 00 \ldots 0| \\
& +b|0 \ldots 01 \ldots 1\rangle\left\langle 1 \ldots 10 \ldots 0\left|+b^{*}\right| 1 \ldots 10 \ldots 0\right\rangle\langle 0 \ldots 01 \ldots 1| \\
& +\sum_{\left\{a_{1}, \ldots, a_{n}\right\}} d_{a_{1} \ldots a_{n}}\left|a_{1} \ldots a_{n} 0 \ldots 0\right\rangle\left\langle a_{1} \ldots a_{n} 0 \ldots 0\right| \\
& +\sum_{\left\{a_{n+1}, \ldots, a_{N}\right\}} g_{a_{n+1} \ldots a_{N}}\left|0 \ldots 0 a_{n+1} \ldots a_{N}\right\rangle\left\langle 0 \ldots 0 a_{n+1} \ldots a_{N}\right|
\end{aligned}
$$


where $|0 \ldots 01 \ldots 1\rangle(|1 \ldots 10 \ldots 0\rangle)$ contains $n$ "0" ("1") and $(N-n)$ "1" ("0"),

$$
c=|\beta|^{2} \prod_{j=1}^{n}\left(1-e^{-\Gamma_{j} t}\right)+|\alpha|^{2} \prod_{l=n+1}^{N}\left(1-e^{-\Gamma_{l} t}\right),
$$

$b$ is the same as was defined in Eq. (18),

$$
d_{a_{1} \ldots a_{n}}=|\beta|^{2} \prod_{j=1}^{n}\left[\overline{a_{j}}\left(1-e^{-\Gamma_{j} t}\right)+a_{j} e^{-\Gamma_{j} t}\right],
$$

and

$$
g_{a_{n+1} \ldots a_{N}}=|\alpha|^{2} \prod_{l=n+1}^{N}\left[\overline{a_{l}}\left(1-e^{-\Gamma_{l} t}\right)+a_{l} e^{-\Gamma_{l} t}\right] .
$$

In Eq. (38) the sum over $\left\{a_{1}, \ldots, a_{n}\right\}$ runs for all possible $a_{1}, \ldots, a_{n} \in\{0,1\}$ except $a_{1}=$ $a_{2}=\ldots=a_{n}=0$, while the sum over $\left\{a_{n+1}, \ldots, a_{N}\right\}$ runs for all possible $a_{n+1}, \ldots, a_{N} \in$ $\{0,1\}$ except $a_{n+1}=a_{n+2}=\ldots=a_{N}=0$, because their contributions are already collected in $c$. It is important to notice the essential difference in Eqs. (16) and (38). In Eq. (16) all the $2^{N}$ diagonal matrix elements are nonzero, whereas this does not so in Eq. (38). In Eq. (38) the diagonal matrix elements $\left|a_{1} \ldots a_{n} c_{n+1} \ldots c_{N}\right\rangle\left\langle a_{1} \ldots a_{n} c_{n+1} \ldots c_{N}\right|$ and $\left|c_{1} \ldots c_{n} a_{n+1} \ldots a_{N}\right\rangle\left\langle c_{1} \ldots c_{n} a_{n+1} \ldots a_{N}\right|$, with $\left\{c_{j}\right\}$ not identically equal to zeros, remain zero all the time. This structural difference between $\rho^{\Phi}(t)$ and $\rho^{\Psi}(t)$ will lead to distinct expressions of the time-dependent negativities that make the disentanglement dynamics of $\left|\Phi_{N}\right\rangle$ and $\left|\Psi_{n, N}\right\rangle$ diverge visibly. As for calculations of negativities they are more subtle for $\left|\Psi_{n, N}\right\rangle$ than for $\left|\Phi_{N}\right\rangle$. Unlike $\left|\Phi_{N}\right\rangle,\left|\Psi_{n, N}\right\rangle$ has two permutationally asymmetric groups of qubits, one group $\left(G_{1}\right)$ contains $n$ first qubits and the other group $\left(G_{2}\right)$ contains the remaining $N-n$ qubits. Therefore, when dealing with a bipartition $k \mid N-k$ we should make clear the "origin" of the $k$ qubits: do all of them come from $G_{1}\left(G_{2}\right)$ or some of them come from $G_{1}$ and the rest from $G_{2}$ ? We may identify that by writing $k=k_{1}+k_{2}$ with $k_{1}\left(k_{2}\right)$ the number of qubits coming from $G_{1}\left(G_{2}\right)$. There are three situations.

First, consider the situation with $1 \leq k_{1} \leq n$ and $k_{2}=0$. The corresponding partially transposed $\rho^{\Psi}(t)^{T_{k_{1}}}$ has the form

$$
\begin{aligned}
\rho^{\Psi}(t)^{T_{k_{1}}=} & c|0 \ldots 00 \ldots 0\rangle\left\langle 0 \ldots 00 \ldots 0|+b| k_{1}\right\rangle\left\langle\overline{k_{1}}\left|+b^{*}\right| \overline{k_{1}}\right\rangle\left\langle k_{1}\right| \\
& +\sum_{\left\{a_{1}, \ldots, a_{n}\right\}} d_{a_{1} \ldots a_{n}}\left|a_{1} \ldots a_{n} 0 \ldots 0\right\rangle\left\langle a_{1} \ldots a_{n} 0 \ldots 0\right| \\
& +\sum_{\left\{a_{n+1}, \ldots, a_{N}\right\}} g_{a_{n+1} \ldots a_{N}}\left|0 \ldots 0 a_{n+1} \ldots a_{N}\right\rangle\left\langle 0 \ldots 0 a_{n+1} \ldots a_{N}\right|
\end{aligned}
$$

with $\left|k_{1}\right\rangle=\left|c_{1} \ldots c_{n} 1 \ldots 1\right\rangle$ and $\left|\overline{k_{1}}\right\rangle=\left|\overline{c_{1}} \ldots \overline{c_{n}} 0 \ldots 0\right\rangle$ where among the $n$ values of $\left\{c_{j} ; j=\right.$ $1,2, \ldots, n\}$ there are $k_{1}$ "1" and $\left(n-k_{1}\right)$ " 0 ". The minimum eigenvalue $\Lambda_{k_{1}}$ of $\rho^{\Psi}(t)^{T_{k_{1}}}$ can be derived as

$$
\Lambda_{k_{1}}=\frac{1}{2}\left(d_{k_{1}}-\sqrt{d_{k_{1}}^{2}+4|b|^{2}}\right)
$$


where

$$
d_{k_{1}}=|\beta|^{2} \prod_{j=1}^{n}\left[c_{j}\left(1-e^{-\Gamma_{j} t}\right)+\overline{c_{j}} e^{-\Gamma_{j} t}\right] .
$$

Next, consider the situation with $k_{1}=0$ and $1 \leq k_{2} \leq N-n$. The corresponding partially transposed $\rho^{\Psi}(t)^{T_{k_{2}}}$ has the form

$$
\begin{aligned}
\rho^{\Psi}(t)^{T_{k_{2}}=} & c|0 \ldots 00 \ldots 0\rangle\left\langle 0 \ldots 00 \ldots 0|+b| k_{2}\right\rangle\left\langle\overline{k_{2}}\left|+b^{*}\right| \overline{k_{2}}\right\rangle\left\langle k_{2}\right| \\
& +\sum_{\left\{a_{1}, \ldots, a_{n}\right\}} d_{a_{1} \ldots a_{n}}\left|a_{1} \ldots a_{n} 0 \ldots 0\right\rangle\left\langle a_{1} \ldots a_{n} 0 \ldots 0\right| \\
& +\sum_{\left\{a_{n+1}, \ldots, a_{N}\right\}} g_{a_{n+1} \ldots a_{N}}\left|0 \ldots 0 a_{n+1} \ldots a_{N}\right\rangle\left\langle 0 \ldots 0 a_{n+1} \ldots a_{N}\right|
\end{aligned}
$$

with $\left|k_{2}\right\rangle=\left|0 \ldots 0 c_{n+1} \ldots c_{N}\right\rangle$ and $\left|\overline{k_{2}}\right\rangle=\left|1 \ldots 1 \overline{c_{n+1}} \ldots \overline{c_{N}}\right\rangle$ where among the $(N-n)$ values of $\left\{c_{j} ; j=n+1, n+2, \ldots, N\right\}$ there are $k_{2}$ " 0 " and $\left(N-n-k_{2}\right)$ " 1 ". The minimum eigenvalue $\Lambda_{k_{2}}$ of $\rho^{\Psi}(t)^{T_{k_{2}}}$ can be derived as

$$
\Lambda_{k_{2}}=\frac{1}{2}\left(g_{k_{2}}-\sqrt{g_{k_{2}}^{2}+4|b|^{2}}\right)
$$

where

$$
g_{k_{2}}=|\alpha|^{2} \prod_{l=n+1}^{N}\left[\overline{c_{l}}\left(1-e^{-\Gamma_{l} t}\right)+c_{l} e^{-\Gamma_{l} t}\right] .
$$

Finally, consider the situation $k=k_{1}+k_{2}$ with both $k_{1} \neq 0$ and $k_{2} \neq 0$. The corresponding partially transposed $\rho^{\Psi}(t)^{T_{k}}$ has the form

$$
\begin{aligned}
\rho^{\Psi}(t)^{T_{k}}= & c|0 \ldots 00 \ldots 0\rangle\langle 0 \ldots 00 \ldots 0|+b| k\rangle\left\langle\bar{k}\left|+b^{*}\right| \bar{k}\right\rangle\langle k| \\
& +\sum_{\left\{a_{1}, \ldots, a_{n}\right\}} d_{a_{1} \ldots a_{n}}\left|a_{1} \ldots a_{n} 0 \ldots 0\right\rangle\left\langle a_{1} \ldots a_{n} 0 \ldots 0\right| \\
& +\sum_{\left\{a_{n+1}, \ldots, a_{N}\right\}} g_{a_{n+1} \ldots a_{N}}\left|0 \ldots 0 a_{n+1} \ldots a_{N}\right\rangle\left\langle 0 \ldots 0 a_{n+1} \ldots a_{N}\right|,
\end{aligned}
$$

with $|k\rangle=\left|c_{1} \ldots c_{n} c_{n+1} \ldots c_{N}\right\rangle$ and $|\bar{k}\rangle=\left|\overline{c_{1}} \ldots \overline{c_{n} c_{n+1}} \ldots \overline{c_{N}}\right\rangle$ where among the $n$ values of $\left\{c_{j}\right.$; $j=1,2, \ldots, n\}$ there are $k_{1}$ " 1 " and $\left(n-k_{1}\right)$ "0" and among the $(N-n)$ values of $\left\{c_{j}\right.$; $j=n+1, n+2, \ldots, N\}$ there are $k_{2}$ " 0 " and $\left(N-n-k_{2}\right)$ " 1 ". The minimum eigenvalue $\Lambda_{k}$ of $\rho^{\Psi}(t)^{T_{k}}$ can be derived as

$$
\Lambda_{k}=-|b|
$$

which turns out independent of both $k$ and $n$.

By definition, the negativities associated with the three above situations are given by

and

$$
\begin{aligned}
& \mathcal{N}_{k_{1}}=2 \max \left\{0,-\Lambda_{k_{1}}\right\}, \\
& \mathcal{N}_{k_{2}}=2 \max \left\{0,-\Lambda_{k_{2}}\right\}
\end{aligned}
$$

$$
\mathcal{N}_{k}=2 \max \left\{0,-\Lambda_{k}\right\}
$$



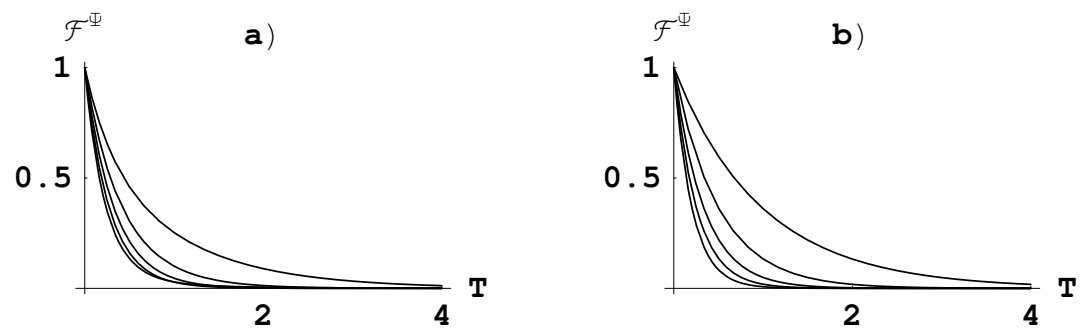

Fig. 5. Fidelity $\mathcal{F}^{\Psi}$, Eq. (55), as a function of $T=\Gamma t$ for $\Gamma_{j}=\Gamma, \gamma_{j}=\gamma$, $\gamma / \Gamma=0.1, N=6$ with different values of $\alpha$ and $n$. a) $\alpha=0.9$ and $n=1,2,3,4,5$ (upwards). b) $\alpha=0.1$ and $n=1,2,3,4,5$ (downwards).

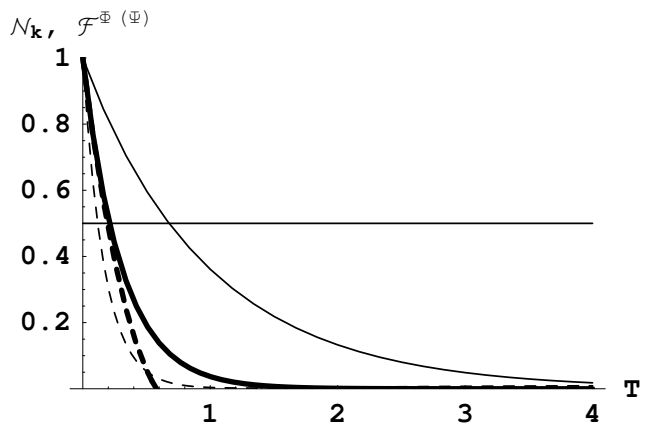

Fig. 6. The largest negativities $\mathcal{N}_{k=N / 2}(T)$, Eq. (20), of state $\left|\Phi_{N}\right\rangle$ (thick-dashed curve) and $\mathcal{N}_{k}(T)$, Eq. (52), of state $\left|\Psi_{n, N}\right\rangle=I^{\otimes n} \otimes\left(\sigma_{x}\right)^{\otimes(N-n)}\left|\Phi_{N}\right\rangle$ (thick-solid curve), in units of their initial value $2|\alpha \beta|$, as functions of $T=\Gamma t$ for $\Gamma_{j}=\Gamma$, $\gamma_{j}=\gamma, \alpha=0.1, \gamma / \Gamma=0.1$ and $N=6$. For the same set of parameters, the entanglement of $\left|\Phi_{N}\right\rangle$ dies suddenly in a finite time, while that of $\left|\Psi_{n, N}\right\rangle$ does asymptotically in an infinite time. The thin-dashed curve is the fidelity $\mathcal{F}^{\Phi}(T)$, Eq. (54), while the thin-solid curve is the fidelity $\mathcal{F}^{\Psi}(T)$, Eq. (55), with the optimal choice $n=1$. The horizontal straight line at 0.5 is just to guide the eye.

respectively. From Eqs. (43), (46), (49) and (50), (51), (52) it immediately follows that all of $\mathcal{N}_{k_{1}}, \mathcal{N}_{k_{2}}$ and $\mathcal{N}_{k}$ tend to zero at the same time as $|b|^{2} \rightarrow 0$. Thus, returning to the expression of $b$, Eq. (18), the condition for all the possible bipartite negativities to vanish, i.e., for $\left|\Psi_{n, N}\right\rangle$ to become completely separable, is simply

$$
e^{-t \sum_{j=1}^{N}\left(\gamma_{j}+\Gamma_{j}\right)}=0 .
$$

This condition (53) means that $\left|\Psi_{n, N}\right\rangle$ never suffers from FTD. Moreover, in the whole range of the involved parameters the entanglement of $\left|\Psi_{n, N}\right\rangle$ decays exponentially at a rate equal to the sum of all the individual decay rates, i.e., additivity of decay rates holds, in transparent contrast to that of $\left|\Phi_{N}\right\rangle$ when $|\alpha|<|\beta|$, as shown in the previous section. This is a remarkable result in the sense that, while the applied local operations do not change the entanglement amount of $\left|\Phi_{N}\right\rangle$, i.e., $\left|\Phi_{N}\right\rangle$ and $\left|\Psi_{n, N}\right\rangle$ possess the same amount 
of entanglement (quantified by any same measure), they cause a dramatic change in the states' disentanglement dynamics.

At this point it is worth noting that, from a practical point of view, what more actually matters is the "short-time" dynamics of disentanglement, i.e., how big is the initial decay rate of the state's entanglement. Also, in view of possible distillation, of significance is the change of the state's fidelity, which is a measure of to what extent the evolved state is close to the initial one. Touching these issues also elucidates the role played by $n$ in the local operator $U(n)$ given by Eq. (36). On the one side, as seen from the condition (53), states $\left|\Psi_{n, N}\right\rangle$ are FTD-free irrespective of a concrete value of $n \in[1, N-1]$. So, to protect $\left|\Phi_{N}\right\rangle$ from FTD, just a single bit-flip, say, of the last qubit (i.e., $n=N-1$ ) suffices. On the other side, however, $n$ proves to play an important role in the evolution of fidelity. For $\rho^{\Phi}(t)$ the fidelity is given by

$$
\mathcal{F}^{\Phi}(t)=\left\langle\Phi_{N}\left|\rho^{\Phi}(t)\right| \Phi_{N}\right\rangle=a|\alpha|^{2}+2 \Re\left(\alpha \beta^{*} b^{*}\right)+b_{11 \ldots 1}|\beta|^{2},
$$

which is of course independent of $n$, while for $\rho^{\Psi}(t)$ it is given by

$$
\mathcal{F}^{\Psi}(t)=\left\langle\Psi_{n, N}\left|\rho^{\Psi}(t)\right| \Psi_{n, N}\right\rangle=g_{11 \ldots 1}|\alpha|^{2}+2 \Re\left(\alpha \beta^{*} b^{*}\right)+d_{11 \ldots 1}|\beta|^{2},
$$

which is sensitive to $n$. So, the strategy is to choose the optimal value $n_{\text {opt }}$ of $n$ such that the decrease of $\mathcal{F}^{\Psi}$ in time is as slow as possible to favor distillation process. This choice depends on the concrete value of $\alpha$. Qualitatively, for $|\alpha|$ close to 1 (i.e., $|\beta|=\sqrt{1-|\alpha|^{2}}$ close to 0$), \mathcal{F}^{\Psi} \simeq g_{11 \ldots 1} \propto \exp [-(N-n) T]$ and thus decays slower for larger $n$ (see $5 \mathrm{a}$ ). In this case the optimal choice is $n_{\text {opt }}=N-1$. In the opposite limit, for $|\alpha|$ close to 0 (i.e., $|\beta|$ close to 1$), \mathcal{F}^{\Psi} \simeq d_{11 \ldots 1} \propto \exp (-n T)$ and thus decays slower for smaller $n$ (see $5 \mathrm{~b}$ ). In this case the optimal choice is $n_{\text {opt }}=1$. Figure 6 visualizes the advantage of $\left|\Psi_{n, N}\right\rangle$ over $\left|\Phi_{N}\right\rangle$ with respect to both entanglement and fidelity evolutions. As recognized from 6 , starting right from $t=0$, not only the entanglement degree of $\left|\Psi_{n, N}\right\rangle$ decays slower than that of $\left|\Phi_{N}\right\rangle$, but also the fidelity of $\left|\Psi_{n, N}\right\rangle$ decays much slower than that of $\left|\Phi_{N}\right\rangle$. In particular, up to $T \simeq 0.66$ the fidelity $\mathcal{F}^{\Psi}$ remains high enough $(>0.5)$ while $\rho^{\Psi}$ is still entangled, enabling a potential entanglement distillation. On the contrary, already after $T \simeq 0.58$ the fidelity $\mathcal{F}^{\Phi}$ is negligibly small and, more seriously, $\rho^{\Phi}$ becomes completely separable due to FTD, preventing any entanglement distillation protocols.

\section{CONCLUSION}

In conclusion, disentanglement dynamics of the multiqubit GHZ-type state $\left|\Phi_{N}\right\rangle=$ $\alpha|0\rangle^{\otimes N}+\beta|1\rangle^{\otimes N}$, each qubit of which is exposed simultaneously to two local damping channels, the phase damping and the amplitude ones, has been studied in detail by means of time-dependent negativities. In contrast to the well-known decoherence of a single qubit, entanglement of $\left|\Phi_{N}\right\rangle$ always vanishes in a finite time. Of outstanding interest is the effect of collective action of different types of noise sources. While in a certain range of the parameters $(|\alpha| \geq|\beta|)$ disentanglement process takes an infinite time under a separate action of either the phase damping or the amplitude damping, FTD turns out to be imperative when both the damping channels are active at the same time, a very pronounced breakdown of additivity of individual decay rates. FTD is obviously an undesirable phenomenon. Whenever it occurs one can by no local means restore the lost nonlocality. Fortunately, 

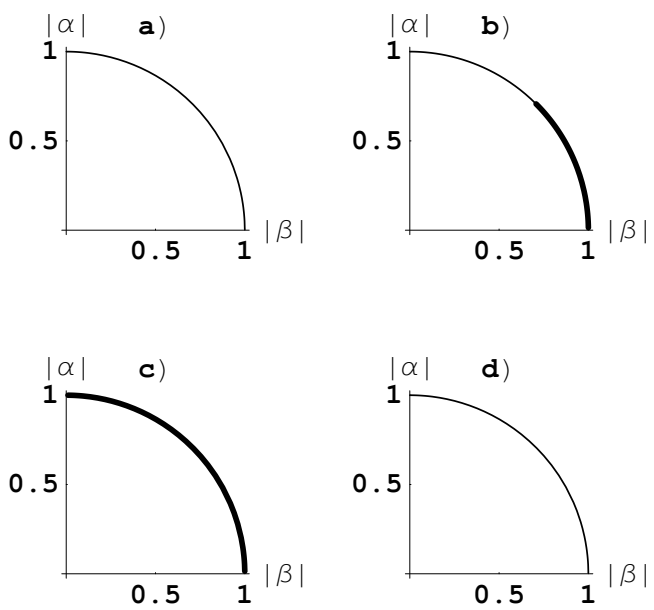

Fig. 7. The state parameter space in terms of $|\alpha|$ and $|\beta|$ (which is a $\frac{1}{4}$-circle of radius 1 except the points $(|\alpha|,|\beta|)=(0,1)$ and $(1,0))$ for a) $\left|\Phi_{N}\right\rangle$ under phase damping alone (no FTD), b) $\left|\Phi_{N}\right\rangle$ under amplitude damping alone (partial FTD), c) $\left|\Phi_{N}\right\rangle$ under phase damping and amplitude damping together (full FTD), and d) $\left|\Psi_{n, N}\right\rangle=I^{\otimes n} \otimes\left(\sigma_{x}\right)^{\otimes(N-n)}\left|\Phi_{N}\right\rangle$ under phase damping alone or amplitude damping alone or both together (no FTD). FTD occurs in the "thick" segment, while asymptotic disentanglement in the "thin" segment. Note that in c) the "thin" segment includes the point with $|\alpha|=|\beta|$.

FTD of $\left|\Phi_{N}\right\rangle$ can be absolutely circumvented without changing its initial entanglement amount by a proper set of local operations: the state $\left|\Psi_{n, N}\right\rangle=I^{\otimes n} \otimes\left(\sigma_{x}\right)^{\otimes(N-n)}\left|\Phi_{N}\right\rangle$ has been shown to always disentangle "normally", i.e., exponentially with the decay rates of all the individual qubits being added for the total rate of the state's entanglement decay. This is true independent of the way of action of damping channels, i.e., no matter they act separately or collectively. Mathematically, such FTD circumvention comes out from the different structures of the reduced density matrices (compare Eqs. (16) and (38)) that in turn yield strongly different forms of the minimum eigenvalues (compare Eqs. (22) with (43), (46) and (49)) of the partial transposes and, thus, of the negativities which govern the states' disentanglement dynamics. Physically, disentanglement is faster for stronger interaction with environments. In the quantum damping model under consideration (see (6)) only qubits in states $|1\rangle$ interact with environments while qubits in states $|0\rangle$ are unaffected. Thus, the more qubits in states $|1\rangle$ the stronger the state-environment interaction. As can be verified, in state $\left|\Phi_{N}\right\rangle$ the probability of finding all the $N$ qubits in states $|1\rangle$ is $|\beta|^{2}$ which is always finite, but in state $\left|\Psi_{n, N}\right\rangle$ such a probability is always zero. In other words, in states $\left|\Psi_{n, N}\right\rangle$ the possible number of qubits being in $|1\rangle$ is always less than $N$ so $\left|\Psi_{n, N}\right\rangle$ interacts with the environments weaker than $\left|\Phi_{N}\right\rangle$ does. It is this fact what is possibly the physical reason of the distinct disentanglement dynamics of the two local-operation invariant states. In this connection, an existing interpretation that under 
the amplitude damping FTD requires the initial population probability of the multiply excited state $|11 \ldots 1\rangle$ to be larger than the population probability of the unexcited state $|00 \ldots 0\rangle[37]$ turns out irrelevant when the phase damping joins the game. The various effects of damping channels and local operations can be viewed diagrammatically in Fig. 7 . The final remark we want to make concerns the value of $N$ which is tacitly treated as greater than or equal to three. However, our results apply to $N=2$ as well, because for $N=2$ the unique bipartition is $1 \mid 1$ for which the defined negativity coincides with concurrence [56]. Experimentally, FTD has been observed in bipartite systems under a single noise source [35-37]. Since quantum technologies are progressing at a quick pace disentanglement dynamics of multipartite systems under the collective action of different types of noise sources could be examined in the future.

\section{ACKNOWLEDGMENT}

This work is supported by a NAFOSTED project NCCB-2009.

\section{REFERENCES}

[1] C. H. Bennett and S. J. Wiesner, Phys. Rev. Lett., 69 (1992) 2881.

[2] C. H. Bennett, G. Brassard, C. Crépeau, R. Jozsa, A. Peres, and W. K. Wootters, Phys. Rev. Lett., 70 (1993) 1895.

[3] A. K. Ekert, Phys. Rev. Lett., 67 (1991) 661.

[4] M. Hillery, V. Buzek, and A. Berthiaume, Phys. Rev. A, 59 (1999) 1829.

[5] P. W. Shor, Proc. 35th Annual Symposium on Foundations of Computer Science, IEEE Computer Society Press (1994) 124.

[6] L. K. Grover, Proc. of the 28th Annual ACM Symposium on the Theory of Computing, ACM Press, New York (1996) 212.

[7] C. Simon and J. Kempe, Phys. Rev. A, 65 (2002) 052327.

[8] W. Dur and H. J. Briegel, Phys. Rev. Lett., 92 (2004) 180403.

[9] A. R. R. Carvalho, F. Mintert, and A. Buchleitner, Phys. Rev. Lett., 93 (2004) 230501.

[10] H. Hein, W. Dur, and H. J. Briegel, Phys. Rev. A, 71 (2005) 032350.

[11] X. S. Ma, A. Wang, X. D. Yang, and F. Xu F, Eur. Phys. J. D, 37 (2006) 135.

[12] S. S. Jang, Y. W. Cheong, J. Kim, and H. W. Lee, Phys. Rev. A, 74 (2006) 062112.

[13] L. Aolita, R. Chaves, D. Cavalcanti, A. Acin, and L. Davidovich L, Phys. Rev. Lett., 100 (2008) 080501.

[14] C. E. Lopez, G. Romero, F. Lastra, E. Solano, and J. C. Retamal, Phys. Rev. Lett., 101 (2008) 080503.

[15] O. Guhne, F. Bodoky, and M. Blaauboer, Phys. Rev. A, 78 (2008) 060301.

[16] Z. X. Man, Y. Xia, and Nguyen Ba An, J. Phys. B: At. Mol. Opt. Phys., 41 (2008) 155501.

[17] Z. X. Man, Y. Xia, and Nguyen Ba An, Phys. Rev. A, 78 (2008) 064301.

[18] T. Yu and J. H. Eberly, Phys. Rev. Lett., 97 (2006) 140403.

[19] W. B. Gao, C. Y. Lu, X. C. Yao, P. Xu, O. Guhne, A. Goebel, Y. A Chen, C. Z. Peng, Z. B Chen, and J. W. Pan, e-print quant-ph/0809.427\%.

[20] S. Bose, V. Vedral, and P. L Knight, Phys. Rev. A 57 (1998) 822.

[21] E. D'Hondt and P. Panangaden, Quantum Inf. and Comp., 6 (2005) 173.

[22] Z. X. Man, Y. Xia, and Nguyen Ba An, Eur. Phys. J. D, 42 (2007) 333.

[23] Nguyen Ba An and J. Kim, Int. J. Quant. Info., 6 (2008) 1051.

[24] J. Preskill, Lecture Notes, Chapter 3, p. 28 (http://www.theory.caltech.edu).

[25] K. Kraus, States, Effects and Operators: Fundamental Notations of Quantum Theory, Springer Verlag (1983) 
[26] T. Yu and J. H. Eberly, Phys. Rev. Lett., 93 (2004) 140404.

[27] L. Jakobczyk and A. Jamroz, Phys. Lett. A, 333 (2004) 35.

[28] T. Yu and J. H. Eberly, Opt. Commun., 264 (2006) 393.

[29] H. T. Cui, K. Li, and X. X. Yi, Phys. Lett. A, 365 (2006) 44.

[30] J. H. Eberly and T. Yu, Science, 316 (2007) 555.

[31] M. Yonac, T. Yu, and J. H. Eberly, J. Phys. B: At. Mol. Opt. Phys., 40 (2007) S45.

[32] I. Sainz and G. Bjork, Phys. Rev. A, 76 (2007) 042313.

[33] M.Ikram, F. L. Li, and M. Zubairy, Phys. Rev. A, 75 (2007) 062336.

[34] X. F. Zhou, Y. Zhang, and G. C. Guo, Phys. Lett. A, 363 (2007) 263.

[35] M. F. Santos, P. Milman, L. Davidovich, and M. Zagury, Phys. Rev. A, 73 (2007) 040305(R).

[36] M. P. Almeida, F. de Melo, M. Hor-Meyll, A. Salles, S. P. Walborn, P. H. Souto Ribeiro, and L. Davidovich, Science, 316 (2007) 579.

[37] A. Salles, F. Melo, M. P. Almeida, M. Hor-Meyll, S. P. Walborn, P. H. Souto Ribeiro, and L. Davidovich, e-print quant-ph/0804.4556.

[38] Z. X. Man, Y. Xia, and Nguyen Ba An, J. Phys. B: At. Mol. Opt. Phys., 41 (2008) 085503.

[39] I. L. Chuang and Y. Yamamoto, Phys. Rev. Lett., 76 (1996) 4281.

[40] L. M. Duan and G. C. Guo, Phys. Rev. Lett., 79 (1997) 1953.

[41] F. Mintert, A. R. R. Carvalho, M. Kus, and A. Buchleitner, Phys. Rep., 415 (2005) 207.

[42] G. Vidal and R. F. Werner, Phys. Rev. A, 65 (2002) 032314.

[43] K. Audenaert, M. B. Plenio, and J. Eisert, Phys. Rev. Lett., 90 (2003) 027901.

[44] D. A. Meyer and N. R. Wallach, J. Math. Phys., 43 (2002) 4273.

[45] T. C. Wei and P. M. Goldbart, Phys. Rev. A, 68 (2003) 042307.

[46] M. Horodeski, P. Horodeski, and R. Horodeski, Phys. Rev. Lett., 80 (1998) 5239.

[47] C. H. Bennett, G. Brassard, S. Popescu, B. Schumacher, J. A. Smolin, and W. K. Wootters, Phys. Rev. Lett., 76 (1996) 722.

[48] C. H. Bennett, D. P. DiVincenzo, J. A. Smolin, and W. K. Wootters, Phys. Rev. A, 54 (1996) 3824.

[49] N. Yamamoto, H. I. Nurdin, M. R. James, and I. R. Petersen, e-print quant-ph/0806.4754.

[50] J. S. Zhang, J. B. Xu, and Q. Lin, e-print quant-ph/0807.2783.

[51] P. W. Shor, Phys. Rev. A, 52 (1995) R2493.

[52] R. Laflamme, C. Miquel, J. P. Paz, and W. H. Zurek, Phys. Rev. Lett., 77 (1996) 198.

[53] A. M. Steane, Phys. Rev. Lett., 77 (1996) 793.

[54] M. P. Plenio, V. Vedral, and P. L. Knight, Phys. Rev. A, 55 (1997) 67.

[55] I. Sainz and G. Bjork, Phys. Rev. A, 77 (2008) 052307.

[56] W. K. Wootters, Phys. Rev. Lett., 80 (1998) 2245. 